\title{
A Modified CDMA/PRMA Medium Access Control Protocol for Voice users in LEO Systems
}

\author{
Abbas Ibrahim, Samir Tohmé \\ Ecole Nationale Supérieure des Télécommunications, \\ Department: InfRes 46, Rue Barrault, 75013 Paris France \\ aibrahim@enst.fr Tel: 0145817552 tohme@enst.fr Tel: 0145817861
}

Key words: LEO satellite channel, CDMA/PRMA, CAC

Abstract: The goal of this paper is to propose a MAC (Medium Access Control) layer to the LEO (Low Earth Orbiting) satellite channel for voice users in order to use efficiently the radio channel bandwidth. This protocol is based on CDMA/PRMA one and adapted to LEO systems. It uses CDMA (Code Division Multiple Access) technique combined with PRMA (Packet Reservation Multiple Access) protocol. A CAC (Connection Admission Control) function that maximizes the number of accepted users with predefined guaranteed QoS (Quality of Service) is then introduced. Furthermore, users traffic control methods are proposed and studied by simulation. The channel parameters are chosen in order to maximize the resource utilization by the proposed protocol.

\section{INTRODUCTION}

Within personal communication system (PCS), the network needs techniques able to handle a wide range of services with different rates. Furthermore the network will provide a global communication service. LEO satellites constellation will probably be an important component of such a network. Voice application using packet mode is one of these services considered as variable bit rate service due to voice activity feature.

The CDMA technique has been chosen in the third generation mobile network IMT2000. While it does not appear to be a single multiple access 
technique that is superior to others in all situations, there are characteristics of spread spectrum waveform that give CDMA certain distinct advantages, especially in mitigating multipath fading of the radio link and interference from other systems [5]. Moreover, in CDMA, integration of circuit-mode and packet-mode traffic requires no special protocol, making the use of packet mode to support voice users easy to realize [6].

Section 2 describes the CDMA technique which, will be used in this paper. The third section describes the system model. Section 4 defines voice application at MAC layer. The fifth section presents the channel model. Section 6 presents the proposed access protocol and the CAC function. The traffic control methods are presented in section 7 and compared in section 8 . Conclusions are presented in section 9.

\section{THE CDMA TECHNIQUE}

In this work, the CDMA/DS (Code Division Multiple Access / Direct Sequence) is used with different frequencies in the neighboring cells (spot beams) [10]. A direct sequence code with a very high rate will be added to the original signal as a signature of the transmitter. The receiver will be able to decode this signature and understand the message. By using different frequency bands in neighboring cells, inter cell interference problem [13] is resolved.

In [11], it is assumed that the performance of a CDMA system is dominated by the bit error ratio (BER) performance and problems related to packet acquisition are ignored. A widely used approximation to determine the BER performance on the CDMA channel is the standard Gaussian approximation (SGA) [9]. Assuming that the MAI (Multiple Access Interference) is Gaussian and using simple correlation receivers, the BER or probability of bit error $P_{e}$ can be obtained from

$$
P_{e}=Q(\overline{S N R})
$$

Where: $Q(x)=\frac{1}{\sqrt{2 \pi}} \int_{x}^{\infty} e^{-u^{2} / 2} d u$

We consider random direct sequences $\left(\operatorname{Pr}\left\{x_{j}=1\right\}=\operatorname{Pr}\left\{x_{j}=-1\right\}=0.5\right)$ where $x_{j}$ is a chip of direct sequence with an arbitrary odd codelength (or spreading factor) $n$. The average signal to noise ratio (SNR) for the ith packet in the case of unequal power reception can be written as 


$$
\overline{S N R}=\sqrt{\frac{P_{i}}{(3 n)^{-1} \sum_{\substack{k=1 \\ k \neq i}}^{K} P_{k}+\frac{N_{0}}{2 T}}}
$$

A system with $K$ simultaneous transmitters is considered with received power levels $P_{j}$ where $(j=1,2, \ldots, k)$, data bit duration $T$ and two-sided spectral density of additive white Gaussian noise $N_{d} / 2$.

In our system no intercell interference will exist because different frequencies are used in neighboring cells. Supposing a perfect power control in the cell, the signal emitted by every transmitter is received by the satellite with $P_{0}$ power level, if we neglect $N_{0}$ [13]

$$
\overline{S N R}=\sqrt{\frac{P_{0}}{\frac{(K-1) P_{0}}{3 n}}}=\sqrt{\frac{3 n}{K-1}} .
$$

Assuming that packets with length $\mathrm{L}$ bits are transmitted over a memoryless binary symmetric communication channel with average probability of data bit success $\left(Q_{e}=1-P_{e}\right)$ and employing a block code, which can correct up to $t$ errors, the packet success probability $Q_{E}$ can be derived from $Q_{E}=\sum_{i=0}^{t} C_{L}^{i}\left(1-Q_{e}\right)^{i}\left(Q_{e}\right)^{L-i}$

And by defining a minor limit of the probability of success we can deduce the maximum number of simultaneous users that can use the channel [7] [2].

\section{SYSTEM MODEL}

The constellation is Iridium like architecture but with different organization of resources. Multibeam antennas are used and each satellite footprint contains a number of spot beams named cells. In each cell uplink and downlink transmissions use two separated radio frequencies, i. e. FDD mode transmission is used. As mentioned in previous section, different frequencies are used in different cells. Assuming that the NCC (Network Control Center) is in one of the gateways. One important function of the NCC is to provide the CAC. The NCC is supposed to have a global view of the network resources. Furthermore, congestion and contention problems are resolved individually at each satellite by using a local control. 


\section{MAC DEFINITION OF VOICE APPLICATION}

At high layer we talk about services supported by the network, for example voice service. At the MAC layer we should talk about capabilities [8]. The mapping between services and capabilities must be defined. In general, service categories are classified to be either real time or non real time. For example in ATM context there are two real time services, CBR (Constant Bit Rate) and VBR-rt (Variable Bit Rate-real time) and three non real time services VBR-nrt, ABR (Available Bit Rate) and UBR (Unspecified Bit Rate). In DiffServ defined with IP (Internet Protocol) there are three differentiated services, premium service for real time applications, assured service and best effort service for non real time applications.

Voice service with voice activity detection can be considered as a real time variable bit rate and can be supported by VBRrt in ATM context and by premium service in IP context. In this article ATM is used and voice service is supported by VBRrt capability and uses AAL2 [12].

\section{CHANNEL MODEL}

The transmission time scale is organized in frames. Each contains a fixed number of slots. The frame rate is identical to the rate of active voice packets. All transmitters transmit their packets such that they arrive at the satellite within the slot boundaries.

The channel is composed of a succession of frames. Each frame contains a number of slots $m$. Each slot can support a maximum number of codes $n$ which are used simultaneously (Figure 1). One special code is reserved for signaling in each slot and does not influence data codes. A code in a slot is named sub slot. The system contains $M=(m \times n)$ sub slots.

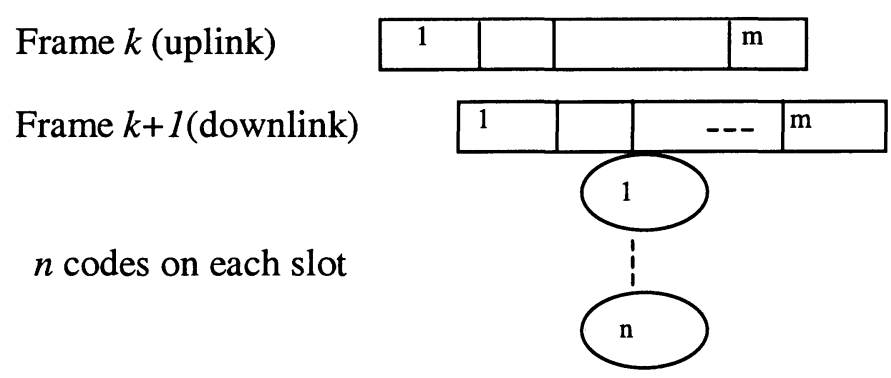

Figure 1. Channel model 
In fact the number of codes used in each slot may exceed $n$ and with acceptable quality. This is due to graceful degradation model. According to this model, there is a non-zero probability of correct reception of any arbitrary number of packet [7]. Moreover, the graceful performance degradation enables the addition of users and the mitigation of errors in protocol operation or in network monitoring without the risk of failure of the system operation.

\section{CHANNEL ACCESS AND ADMISSION FUNCTION}

For voice users there is two important activities: the arrival of a new voice user and the arrival of a voice talkspurt. When a user arrives to the system, he transmits a bandwidth demand request packet. After successful reception of this request, the satellite forwards the request to the NCC and responds to the terminal.

In case the NCC has accepted the demand, the terminal $a$ has then to contend on slot $i$ to transmit his first packet. He does, first, a Bernoulli experiment with $P_{i a}$ probability calculated in relation with the information broadcast by satellite, then he decides to send or not. He switches from contention to reservation mode as soon as he realizes that he sent on a convenient slot. At the beginning of each talkspurt the terminal repeats the process.

Notice that some packets may be lost at the beginning of each talkspurt. They are added to the set of dropped packets. This set is composed of dropped packets at the beginning of talkspurts and erroneous packets due to CDMA interference.

In contrast to PRMA defined in [3] and PRMA-HS (Hindering State) presented in [4], terminals do not classify slots as either "reserved or available" because the channel access for contending terminals is governed by time-varying permission probability. Another difference with PRMA is the CAC function. In our case a direct and simple one is used. A request for setting up a new voice connection will be accepted if there are less than $M \times f$ accepted conversations in the cell, where $M$ is the number of sub slots in the channel and $f$ is a factor higher than one and depends on the access protocol used. 


\section{THE TRAFFIC CONTROL}

In order to fully utilize the channel capacity while maintaining the quality of voice service acceptable, probability of allowing transmission in each slot $i$ for such a user $a, P_{i a}$, needs to be dynamically updated according to the information about the system state available within the satellite and broadcast to users.

In contrast with [1], $P_{i a}$ is calculated in the mobile station and not in the satellite, that makes it more flexible and simplifies the satellite design. In fact, $P_{i a}$ is set according to the number of voice users who used the slot $i$ in the previous frame. The purpose of this function is to control the total number of users in every slot, such that, the throughput is maximized without exceeding the loss limit, which means, without exceeding the total number of codes allowed to be used simultaneously.

Assume that the satellite, by using its local memory, knows the number of simultaneous codes used in each slot in the last frame $M I_{i}$. We have then to choose the function which calculates the probability of sending on a slot $i$, this function depends on $M I_{i}$ and should minimize the probability of collision on the slot, that means should distribute users on slots as uniform as possible. Two methods to calculate these probabilities are presented.

1. The first one is the following. Taking into account that each user knows the number of codes used on each slot in the previous frame, user calculates permission probability by:

If $M I_{i}<n$ then $P_{i a}=p-\operatorname{tg}(\alpha) \times M I_{i}$,

Otherwise $P_{i a}=\max \left(p-\operatorname{tg}(\alpha+\beta) \times M I_{i}, 0\right)$.

Where $n$ is the spreading factor, $\operatorname{tg}()$ is the tangent function, $p$ is the initial probability, $\alpha$ and $\beta$ are the slopes of the two linear segments represented by the two functions above. These parameters should be chosen to maximize the throughput.

In [1], authors have studied an efficient protocol which uses this computation but with different strategy. In this protocol, user has to be acknowledged to switch to reservation mode. If user is not acknowledged he must retransmit. He can retransmit until the waiting time exceeds " $D_{\max }=$ $20 m s$ " which is the threshold of waiting time for voice user. For satellite communication, this is impossible because of the high round trip time which is comparable with $D_{\max }$. Two variations are introduced.

The first is at the protocol scale: instead of waiting the acknowledgement to switch to reservation mode, the user waits the slot state broadcast by satellite. If the number of codes used on this slot is less than the spreading factor, the user continues the usage of the same slot, if not he must recontend. 
The second is for values of $p, \beta$ and $\alpha$, In [1] the values chosen are " $p=$ $0.3, \alpha=0.007$ and $\beta=0.1$ ". These values are bad in LEO context and produces high dropping probability at the beginning of each talkspurt. The reason is that in LEO system the packet can not wait more than one frame time and the user tries to send this packet on slots dedicated for voice in only one frame time. The new choice is then " $p=0.8, \alpha=0.02$ and $\beta=0.2$ ".

2. The second method is inspired from the fact that, in CDMA used, the maximum number of simultaneous codes which can be used with acceptable quality of service (loss $<0.01$ ) exceeds the spreading factor. So we define two thresholds of control: the spreading factor $n$ and the maximum number of accepted codes in a slot $s$. The method is as follow:

If the value of $n$ is larger than $M I_{i}$, a voice user, in order to begin a talkspurt, uses a code in the slot $i$ with probability $\quad P_{i a}=1$.

If $n \leq M I_{i} \leq s$, then $P_{i a}=n /\left(M I_{i} \times U\right)$

Where $U$ is a constant higher than one and must be chosen to have the maximum efficiency of the protocol.

In other cases $\quad P_{i a}=0$.

After sending the first packet, the user switches to reservation mode if the number of codes used on this slot is less than $s$. In the other case he must recontend.

\section{SIMULATION RESULTS}

These simulations compare different methods presented in section 7 with different parameters of channel. The simulator tool (NS Network Simulator) is used to define CDMA/PRMA channel supporting the proposed protocol. The LEO system used is Iridium like architecture with multibeam technology. In each beam we have 24 frequency carriers and each one has a downlink bandwidth equal to uplink bandwidth equal to $512 \mathrm{~Kb} / \mathrm{s}$. Gateway (GW) access bandwidth is $155,52 \mathrm{Mb} / \mathrm{s}$. ATM cells are used to support voice packets and therefore data field is 48 bytes length and correction field is 10 bits length. Voice rate in the "on" state is $8 \mathrm{~Kb}$ and channel parameters are assigned two different values:

In the first case, the following parameters are used

- number of slots $=8$

- spreading factor $=n=512 \mathrm{~Kb} / 8 \mathrm{~Kb} / 8=8$

- accepted codes $=s=10$ (from computation in section 2 with $Q_{E} \geq$ 0.99)

The second case considers the following values:

- number of slots $=4$

- spreading factor $=n=512 \mathrm{~Kb} / 8 \mathrm{~Kb} / 4=16$ 
- accepted codes $=s=20$ (from computation in section 2 with $Q_{E} \geq$ 0.99)

The mean time of the state $\mathrm{ON}\left(T_{O N}\right)$ is set to one second and the one of OFF state $\left(T_{O F F}\right)$ is fixed at 1.35 seconds. The simulation run time is such that each user is in conversation during $3 \mathrm{mn}$, which is equal to the mean time of voice connection. That necessitates many hours of simulation time and makes the simulation confidential.

Figure 2 compares the two control methods for the two choices of channel parameters. This comparison leads to determine the maximum number of acceptable users in each case. This number determines the limit used by $\mathrm{CAC}$ function. As this number augments as the efficiency of the protocol increases:
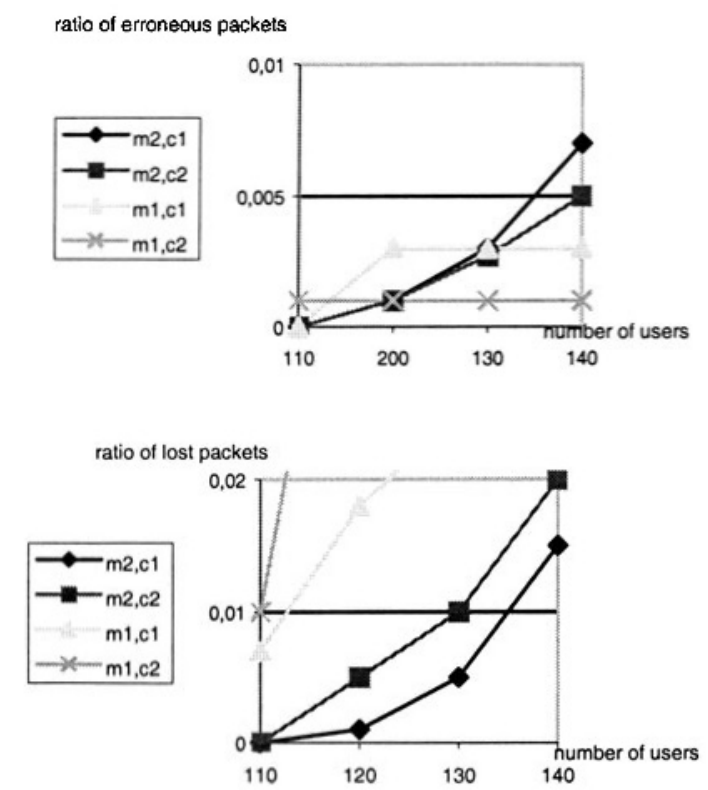

Figure 2. Comparison of methods and choices (mi, cj = method "i" for choice "j")

The second graph in Figure 2 is the significant one and proves that the second method with first choice gives the best results. With the first method the first choice is better. In the first graph we see the ratio of erroneous packets due to CDMA interference only. In this graph we notice a lower error ratio in the first method because the limit of accepted users on slots have not been attained in the first method due to a high drop probability. Anyway, the main criterion of performance is the total loss ratio. To clarify this discussion Figures 4 and Figures 5 present distribution of users in different cases. 
Figure 4 shows that the first method is acceptable and the limit of users in a slot (10 users) is attained. On the contrary, the limit (20 users) is not attained in the second choice because of a high drop probability.

Figure 5 illustrates that the second method does significantly improve the utilization of resources and the limit of users on slots is frequently attained and especially for the first choice. This improvement allows augmenting the number of accepted users up to 135 for the first choice as presented in figure 2. This number represents the threshold of CAC function. This threshold varies from 110 users to 135 users and $f$ defined in section 6 varies from 1.7 to 2.1 and its value depends on the control method.

An important performance issue is the multiplexing efficiency relative to perfect statistical multiplexing. We define the multiplexing efficiency factor as: $\quad \mu=M_{0.01} \times \delta / m \times n$.

Where $M_{0.01}$ is the number of simultaneous conversations supported with a loss probability less than 0.01 . $\delta$ is the voice activity factor given by $\delta=$ $T_{O N} /\left(T_{O N}+T_{O F F}\right)$ and $(m, n)$ are the slot number and the spreading factor respectively. Table 1 lists this factor for different cases as well as for PRMA and PRMA-HS protocols. This table shows the enhancement of multiplexing efficiency for the proposed protocol with a good choice of the channel parameters.

Table 1: multiplexing efficiency comparison

\begin{tabular}{|c|c|}
\hline $\begin{array}{c}\text { Protocol, choice, } \\
\text { method }\end{array}$ & $\begin{array}{c}\text { Multiplexing efficiency } \\
\text { factor (packets/slot) }\end{array}$ \\
\hline CDMA/PRMA, 1, 1 & 0,73 \\
\hline CDMA/PRMA, 2, 1 & 0,75 \\
\hline CDMA/PRMA, 1, 2 & 0,9 \\
\hline CDMA/PRMA, 2, 2 & 0,87 \\
\hline Classical PRMA & 0,67 \\
\hline PRMA-HS & 0,73 \\
\hline
\end{tabular}

Finally, Figure 3 presents the effect of channel parameters on the efficiency of the protocol. These parameters can be represented by the spreading factor. When the spreading factor varies between 64 and 1, the system varies from pure CDMA to pure PRMA 


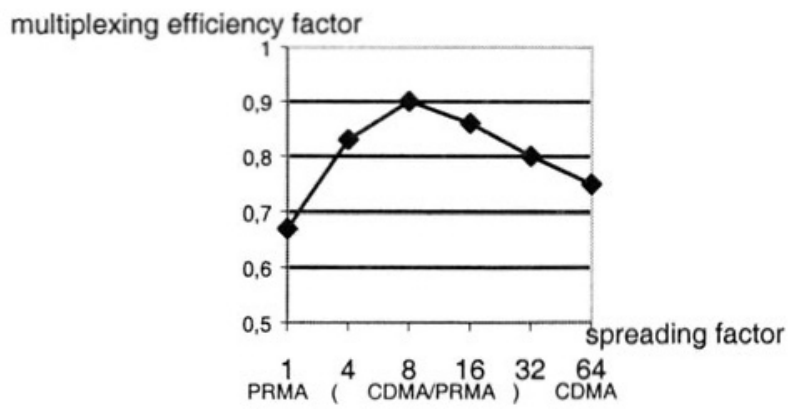

Figure 3. comparison of different channel parameters (second method)

number of users on

slots

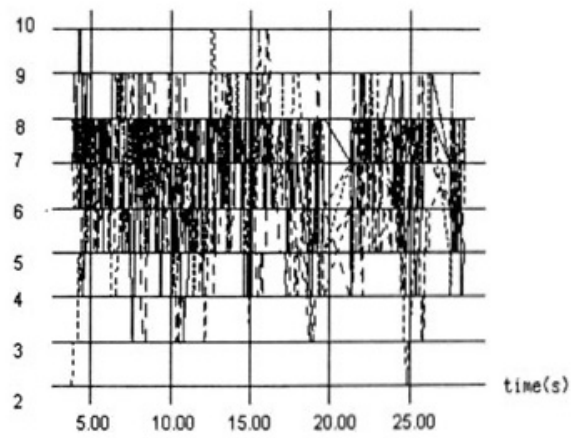

number of users on

siots

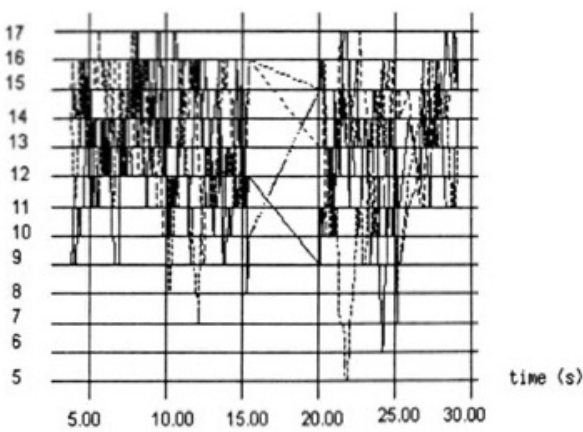

Figure 5: Distribution of 135 users on slots (first and second choices, second method)

\section{CONCLUSION}

In this paper, a modified CDMA/PRMA protocol is proposed in order to provide access to radio channels in LEO systems. Control algorithms which calculate dynamically the permission probability are then proposed. Choices of channel parameters are presented in order to choose the best one. The comparison of the control methods with various choices gives numerical results which, find out the best method and the best choice. These results illustrate how very high multiplexing efficiency can be achieved by using two thresholds control algorithm with a spreading factor equal to 8 . This choice improves the proposed protocol in term of the efficiency of using resources and in term of the increase of system capacity. 


\section{REFERENCES}

[1] A. E. Brand and A. Hamid Aghvami "Performance of Joint CDMA/PRMA Protocol for Mixed Voice Data Transmission for Third Generation Mobile Communication" IEEE J. on Select. Areas. in Comm. December 1996.

[2] C. LI and R. D.Gitin "Multicode CDMA Wireless Personal Communications Networks" IEEE ICC'95.

[3] D. J. Goodman, R.A. Valenzuela, K.T.Gayliard and Ramamursh "Packet Reservation Multiple Access for Local Wireless Communications" IEEE Trans. on Comm. August 1989.

[4] D. Re Enrico, Romano Fantacci, Giovanni Giambene, and Walter Sergio. "Performance Analysis of an Improved PRMA Protocol for Low Earth OrbitMobile Satellite Systems" IEEE Trans. on Vehicular Technology, MAY 1999

[5] G. Evaggelos., Yu-Wen CH., Wen-Bin Y. "Optimal Strategies for Admitting Voice and Data Traffic in Networks of LEO Satellites using CDMA" Wireless Networks 2(1996) 315-328.

[6] J. Abbas. "Low Earth Orbital Satellites for Personal Communication Networks". Artech House. Boston, London.

[7] K. S.Gilhousen, I.M. Jacobs, R. Padavoni and L.A. Weaver "Increased Capacity using CDMA for Mobile Satellite Communication". IEEE J. on Select. Areas. in Comm. 8 (4) 1994.

[8] K. Heba and T. Samir "Resource Allocation And Connection Admission Control in Satellite Networks" IEEE J. on Select. Areas in Comm. February 1999.

[9] M. Gerard and M. Bousquet "Satellite Communication System" third edition. WILLEY 1998.

[10] M. B. Pursley, "Performance Evaluation for Phase Coded Spread Spectrum Multiple-Access Communication part-I: system analysis" IEEE Trans. on Comm. August 1977.

[11] N. D. Wilson, R. Ganesh, K. Joseph, and D. Raychaudhuri, "Packet CDMA versus Dynamic TDMA for Multiple Access in an Integrated Voice Data PCN" IEEE J. on Select. Areas in Comm. August 1993.

[12] K. Sriram, Y. terng wang "Voice Performance Using AAL2 and Bit Dropping Performance and Call Admission Control" IEEE J. on Select. Areas in Comm. January 1999

[13] V. Andrew J. "CDMA Principles of Spread Spectrum Communication" AddisonWelsey 1995. 